\title{
Robot Path Planning Agent for Evaluating Collaborative Machine Behavior
}

\author{
Ma Baoping ${ }^{1}$, Yang Haoyue ${ }^{1}$, Wei Jingcheng ${ }^{2}$, Meng Qingmin ${ }^{2}$ \\ ${ }^{1}$ School of Electrical Engineering and Automation, Nanjing Normal University \\ ${ }^{2}$ School of Communication and Information Engineering, Nanjing University of Posts and \\ Telecommunications, Nanjing, China
}

\begin{abstract}
We first review the literature on machine behavior which may contain the interaction between machines, and then discuss the methodology and the related case involving collaboration between robots to instantiate the concept with AI empowerment. The navigation case aims to a security application with autonomous control and roadside agent embedded in a mobile edge computing structure. In the studied navigation based on the artificial potential field method, robots need to use position information to calculate the moving direction frequently. In the case of high motion speed as well as GPS positioning error, the path trajectory may show a sharp change of direction. In order to mitigate the trajectory oscillation, this paper proposes a path planning design where training process and motion direction prediction are integrated by using artificial neural network. The auxiliary navigation agent near multiple obstacles can first extract the past movement information of the robot and then determines whether there is a serious path jitter event. Computer simulation analysis shows that the combination of autonomous control and cooperative behavior can effectively reduce the path jitter so as to achieve a fast and safe path planning.
\end{abstract}

Keywords: machine behavior, mobile robots, navigation, path planning, artificial potential field, artificial neural network

\section{Introduction}

With the development of artificial intelligence (AI) and cloud computing, the use of AIdriven machines and mobile robots will gradually become more frequent. In the next few years, human society will usher in the intelligent era of human-machine interaction and machinemachine collaboration. The AI empowerment interaction and collaboration is attracting the attention of some researchers. As Rahwan described in the latest work [1], the current academic community is at a crossroads in understanding AI-enabled machine systems. Before this point of intersection, people usually used all kinds of traditional science to study how machines imitate people to learn. But after this point of intersection, new methodologies will be needed to study a new field - machine behavior. The new field includes not only computer science disciplines but also insights from other disciplines. Therefore, people need multidisciplinary knowledge to deeply understand and design the machine systems. In the following, we first discuss the related literature on machine behavior, and then study a security case with mobile edge computing: cooperative path planning for mobile robots. 


\section{Literature Review}

A. Machine behavior and AI cloud computing

As a new network architecture and an entity at the edge of the cellular network, mobile edge computing can not only reduce the pressure on the cloud by processing edge data, but also facilitate more computing, storage and analysis functions for smaller devices. By collecting, storing, and analyzing data from local machines, mobile edge computing entities can become observers and coordinators of machine behavior, facilitating control and security applications. The applications may include mobile robot motion and behavioral awareness, adaptive control of walking robots, detection of behavior-based malicious applications and more. Therefore, we believe that AI-enabled mobile edge computing is a kind of AI cloud computing, which is a reliable solution to study the behavior of machines.

The complex control behavior of online learning autonomous mobile robots is one of the current research topics [2]. The study of the motion patterns of socially conscious robots needs to follow three main directions: behavioral prediction, reinforcement learning and behavioral cloning [3].

There has been a lot of work on mobile edge computing that integrates machine learning. In the work [4], various deep learning algorithms are introduced into the intelligent transportation system. The results show that the intelligent transportation system combining the edge analysis architecture and the deep learning algorithm can realize a reliable, safe and truly intelligent traffic environment. The literature [5] proposed a mobile edge computing model based on deep learning, which can efficiently detect malicious applications at the edge of cellular networks. In addition, there is also document discussing the security challenges faced by edge computing, such as how to ensure privacy and security when mobile devices belonging to users who do not trust each other participate in public computing. The work in [6] proposed a new lightweight framework based on edge computing for CNN feature extraction of mobile sensing. The framework is able to significantly reduce the latency and overhead of end devices while protecting privacy.

\section{B. Path planning for mobile robots}

Since the methodology of machine behavior is in its infancy, we will focus on a specific case of machine behavior, namely collaborative path planning for mobile robots.

For mobile robots, path planning and obstacle avoidance have become the focus of research. Path planning refers to the optimal path selection of robots from the starting point to the target point through positioning devices and sensors. To solve the problem of collision-free and fast addressing of mobile robots in an unknown environment, many scholars have proposed efficient algorithms, such as artificial potential field(APF) method [7], A* algorithm [8], RRT algorithm [9], genetic algorithm [10] and so on. Among them, the artificial potential field method is a local path planning algorithm, which has the advantages of simple principle and small computation.

The traditional artificial potential field (TAPF) method has the problem of the local minimum trap. Many researchers have come up with their own solutions to this problem. A new potential function to replace the traditional artificial potential field method is proposed in [11]. By adding virtual obstacles, the robot could avoid falling into local traps. In [12], an adaptive repulsive potential function is studied, which was combined with the rolling window 
method to solve the local minimum problem. Literature [13] proposed an improved artificial potential field method based on connectivity analysis. Through analyzing the connectivity of obstacles, the scheme obtains a feasible solution domain according to the geometric topology. The velocity factor was added in the potential field function so that users could avoid dynamic obstacles and reach dynamic targets. An improved obstacle potential field function model is considered in [14], which takes into account the size of the robot and the obstacle and adaptively changes the weight of the obstacle potential field function to make the robot escape from the local minimum.

The above literature considers some cases the robot has complete knowledge of its environment. However, there are potential cases that the robot moves across the complex potential field area at a big pace of movement and positioning errors occur due to poor radio propagation. Relevant researchers have shown that when high motion speed and GPS positioning errors are taken into account, the trajectory of the robot is likely to shake violently [15-16]. As shown in Figure 1, when the robot moves between obstacles or in the aisle, the sharp change of direction problem is extremely serious. There are only several works and solutions to this problem. The presence of obstacles and narrow aisle and the oscillation problem of potential field methods are studied in [15] where a modified version of Newton's method is proposed. Compared with the standard gradient descent method, the use of the modified Newton method can greatly improve the performance of the system and reduce the path jitter. In work of [16] the traditional gradient descent technique is compared with the second-order optimization method, and its results show that the Levenberg-Marquardt algorithm can improve the jitter problem and generate smoother trajectory with fewer steps.

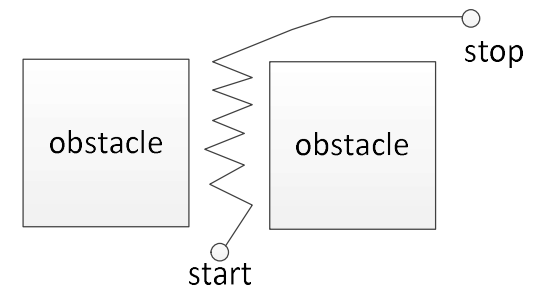

Figure 1. Trajectory oscillation in the case of large moving steps and GPS position errors

\section{Methodology}

Part of the research method process will be explained here. Taking the collaborative application in machine behavior as an example, the purpose of synergy between the mobile robot and the navigation agent is to enhance security. A general overview of the research methodology is shown in Figure 2.

a). data collection and storage

The motion state data collected by the sensors of the cooperative mobile robot is transmitted to the mobile edge server by the wireless network for storage.

b). machine behavior monitoring

The navigation agent will analyze this data online or offline. Through analysis, the agent will determine whether the robot's behavior is malicious or benign.

c). data preprocessing

The data obtained by the agent is often dirty and requires further processing.

d). learning, classification and prediction 
The processed data and the appropriate algorithm are used for modeling to realize the decision of the future behavior of the robot with malicious behavior.

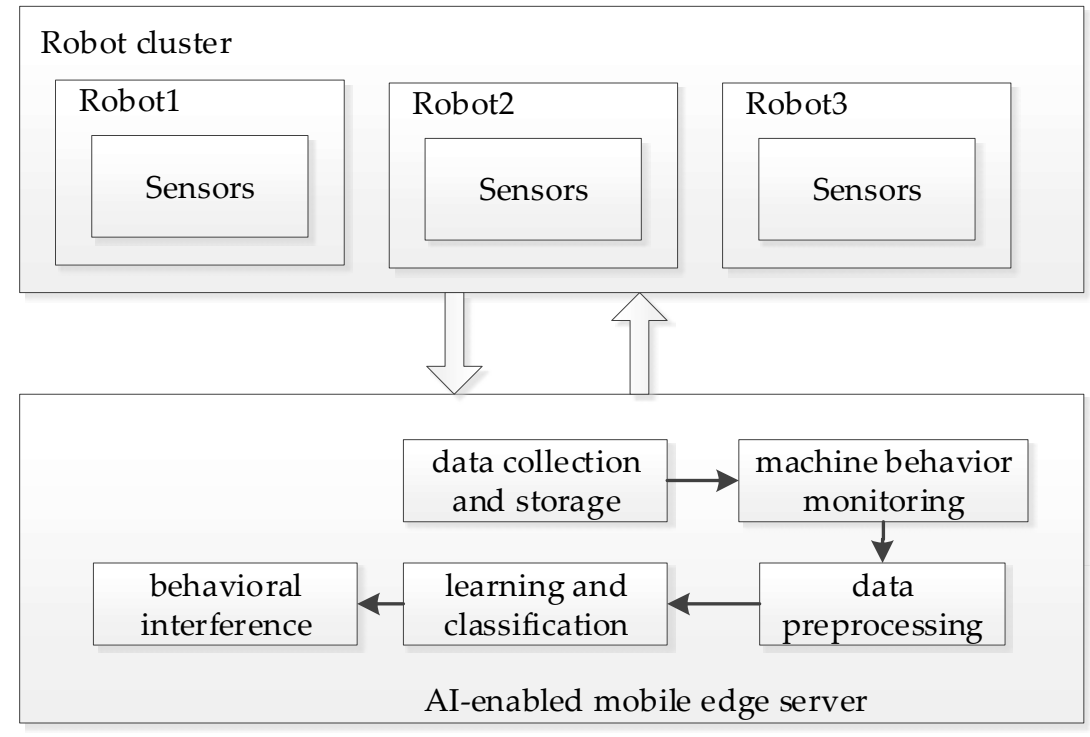

Figure 2. Conceptual diagram of collaborative application in machine behavior

In particular, this paper considers the application of machine collaboration and safe navigation. The scenario considers the situation of large moving steps and the existence of GPS position errors and the resulting trajectory oscillation problems. At this point, the robot may need to use external systems and external calculations to improve the performance of autonavigation. Here we refer to the external network entity as the agent, and the external calculation is considered as a type of mobile edge computing. The contribution of this paper is to propose a path planning agent and consider the design of a machine learning algorithm that can use small test samples. The robotic path planning scheme is presented which combining artificial neural network (ANN) and TAPF to help the robot obtain a smoother moving path.

\section{TAPF}

TAPF is actually to establish a virtual potential field in the environment. The target point will generate a gravitational field on the robot, which will attract the robot to move towards it. While the obstacle will form a repulsive field, which will form a repulsive force on the robot to prevent the collision between the robot and the obstacle. The superposition of the gravitational field and repulsive field guides the robot to move towards the target point while avoiding obstacles [7].

It is more important to construct the gravitational field and the repulsive field in TAPF. The gravitational field function and the repulsive field function are shown in formula (1) and formula (2) [17]:

$$
\begin{gathered}
U_{a}(X)=\frac{1}{2} \xi\left(d\left(X, X_{g}\right)\right)^{2}, \\
U_{r}(X)= \begin{cases}\frac{1}{2} \eta\left(\frac{1}{d\left(X, X_{o}\right)}-\frac{1}{d_{0}}\right)^{2}, & d\left(X, X_{o}\right) \leq d_{0}, \\
0, & d\left(X, X_{o}\right)>d_{0}\end{cases}
\end{gathered}
$$


where, $\xi$ and $\eta$ respectively represent the gravitational gain coefficient and repulsive gain coefficient, $X, X_{g}$, and $X_{o}$, respectively represent the geographic position of the robot, target point, and obstacle. $d(\cdot)$ is the distance calculation function, and $d_{o}$ represents the influence range of the obstacle. Only when the robot enters the influence range of the obstacle the influence of the repulsive force field should occur.

Assume that there is a GPS positioning error $X_{N}$, which obeys the normal distribution with mean $\mu$ and variance $\sigma^{2}$, i.e. $X_{N}: N\left(\mu, \sigma^{2}\right)$. In this case, the position obtained by the robot is not the real geographical position $X$, but the biased geographical position $\bar{X}=X+X_{N}$. Then, the gravitational field function of formula (2) needs to be modified into formula (3),

$$
U_{a}(\bar{X})=\frac{1}{2} \xi\left(d\left(\bar{X}, X_{g}\right)\right)^{2}
$$

Since the distance between the robot and the obstacle is obtained by laser ranging, which is the distance value without noise pollution, formula (2) can still be used to express the repulsion force field function without modification.

According to the potential field function constructed, the gravitational force $\vec{F}_{a}(X)$ and repulsive force $\vec{F}_{r}(X)$ on the robot can be obtained, as shown in formula (4) and formula (5),

$$
\begin{gathered}
\vec{F}_{a}(\bar{X})=-\nabla U_{a}(\bar{X})=-\xi d\left(\bar{X}, X_{g}\right) \frac{\partial d\left(\bar{X}, X_{g}\right)}{\partial \bar{X}}, \\
\vec{F}_{r}(X)=-\nabla U_{r}(X)= \begin{cases}\eta\left(\frac{1}{d\left(X, X_{o}\right)}-\frac{1}{d_{0}}\right) \frac{1}{d\left(X, X_{o}\right)^{2}} \frac{\partial d\left(X, X_{o}\right)}{\partial X}, & d\left(X, X_{o}\right) \leq d_{0}, \\
0, & d\left(X, X_{o}\right)>d_{0}\end{cases}
\end{gathered}
$$

where $\nabla U_{a}(\bar{X})$ and $\nabla U_{r}(X)$, respectively represent the gravitational field gradient and the repulsive field gradient.

According to the force analysis on the robot in Figure 2, the resultant force $\vec{F}_{j}(X, \vec{X})$ on the robot can be expressed as

$$
\vec{F}_{j}(X, \bar{X})=\vec{F}_{a}(\bar{X})+\vec{F}_{r}(X)
$$

Referring to Figure 3, due to the positioning error, the robot's attraction is changed from $\vec{F}_{a}(X)$ to $\vec{F}_{a}(\bar{X})$. Finally, the resultant force deviated from the optimal movement direction. When the step size of the robot is large, the path jitter phenomenon is remarkably intensified.

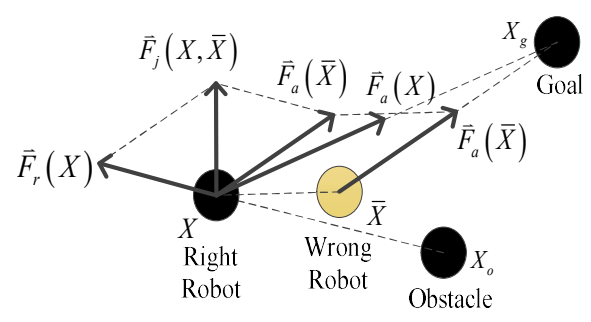

Figure 3. Force analysis diagram of the robot 


\section{Safety Planning of Artificial Potential Field Combined with ANN}

As mentioned above, many current studies on APF-based algorithms are based on the premise of ignoring the robot position error. However, when relatively large moving speed and the positioning error are taken into account in the process of robot movement, the moving route of the robot will have a serious jitter phenomenon, as shown in figure 1, which will greatly affect the path planning speed of the robot. In this paper, the artificial neural network is combined with TAPF to help the robot quickly get rid of the region with severe jitter.

In order to solve the jitter problem, two problems need to be considered first: whether the robot has serious jitter and how to leave the jitter area. Considering the limited computing power of the robot, our specific implementation is to offload the computing task to the agent. Considering the real-time nature of autonomous navigation, we also need to consider a mobile edge calculation using small samples, such as shallow neural networks. Of course, the proposed solution needs to upload the position and direction information to the auxiliary navigation system.

\section{A. Dithering judgment}

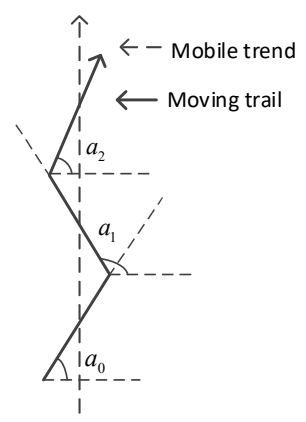

(a) A slight shaking

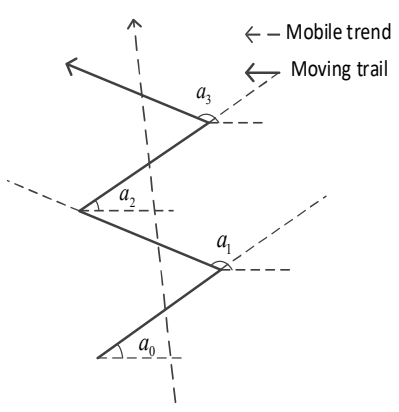

(b) Severe vibration

Figure 4. Comparison of direction angles when different jitter occurs.

As for how to judge whether there is a severe jitter, we can start from the movement history data of the robot. First of all, the direction angle of the $i+1$ times movement (the $i+1$ footstep) of the robot is defined as $a_{i}$. The absolute difference of the direction angle of two adjacent movements of the robot is $\beta_{i}$ as shown in formula (7),

$$
\beta_{i}=\left|a_{i}-a_{i-1}\right| .
$$

Two jitters with different levels are shown in Figure 4. It can be observed that when the shift direction is small, $\beta_{i}$ is small; when the shift direction is large, $\beta_{i}$ is relatively large.

For the $i+1$ movement of the robot, the agent extracts the direction angle sequence $A_{i}=\left[a_{i}, a_{i-}\right.$ $\left.{ }_{1}, \cdots, a_{i-N+1}\right]$ for the first $N$ movements of the robot and calculates the sequence $B_{i}=\left[\beta_{i}, \beta_{i-1}, \cdots \beta_{i-}\right.$ $\left.{ }^{N+2}\right]$, i.e., the absolute difference between two the adjacent directional angles. When all elements in sequence $\beta_{i}$ are greater than the absolute difference threshold of trajectory direction $\beta_{c}$, it can 
be judged that the robot has serious trajectory jitter, which can be expressed as formula (8)

$$
\phi_{i}=\left\{\begin{array}{l}
1, \quad \forall \beta_{j} \in B_{i}, \beta_{j} \geq \beta_{c} \\
0, \quad \exists \beta_{j} \in B_{i}, \beta_{j}<\beta_{c}
\end{array}, i \leq j \leq i-N+2,\right.
$$

where, $\phi_{i}$ represents whether the robot has serious jitter before the first movement, and the value is 1 or 0 . $\phi_{i}=1$ indicating that the robot's trajectory has serious jitter, and $\phi_{i}=0$ indicating that the robot's trajectory has no serious jitter.

\section{B. Prediction of future motion direction}

When there is severe jitter, in order to quickly leave the jitter area, we introduce an ANN algorithm to predict the movement direction or trend of the robot in a period of time in the future. In the following period of time, the robot abandons TAPF algorithm and advances a certain distance according to the predicted direction.

ANN is a network structure composed of many neurons, including the input layer, hidden layers and output layers 18. The neurons between the two adjacent layers are connected with each other and have excellent nonlinear approximation ability. Due to its strong performance, it has been widely used in the fields of pattern recognition, medicine and biology 19. Backpropagation algorithm (BP) is a relatively popular ANN algorithm at present. It is composed of the forward propagation of information and the backpropagation of error, and the ANN model with the high accuracy can be obtained by constantly narrowing the error 21 . In order to make the trained neural network have better performance and prediction ability, it is necessary to select data that can represent the future movement trend of the robot as the input feature.

In this work, the moving direction angle information $A_{i}$ before the $i+1$ movement of the robot and the distance information $D_{i}=\left[d_{i}, d_{i-1}, \cdots, d_{i-N+1}\right]$ between the robot and the target point are used as the sample characteristics, where $d_{j}(i \leq j \leq i-N+1)$ represents the distance between the robot and the target point after the $j$ movement, and thus constitutes the sample $S_{i+1}=\left[A_{i}, D_{i}\right]$ for the trained ANN. In addition, by observing Figure 4, it can be found that even if the robot is in a trajectory oscillation state, there is a moving trend direction (as shown by the dotted arrow). We define the movement trend direction of the robot before the $i+1$ movement as $P_{i+1}$, which is taken as the label of sample $S_{i+1}$ and used as the $i+1$ movement direction of the robot. Therefore, we build and train the ANN model containing one hidden layer to predict the movement trend of the robot.

\section{Combined scheme}

In order to solve the serious jitter problem of robot movement trajectory in the case of high moving speed as well as GPS positioning error, the robot path planning scheme we studied combines ANN and TAPF, and the scheme flow is shown in Figure 5. 


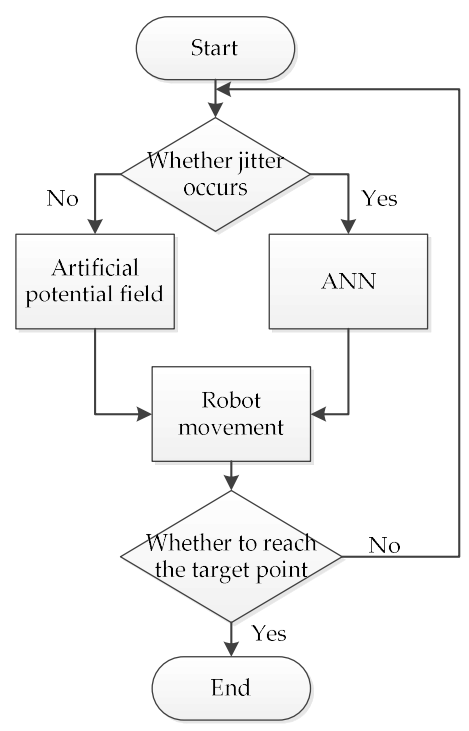

Figure 5. Program flow chart

In this scheme, the robot mainly relies on the artificial potential field method for path planning, but considering the high-speed requirement and GPS positioning error, the robot is prone to serious path jitter, so the ANN algorithm is needed to reduce the occurrence of jitter. The process of the program can be summarized as follows:

1). first of all, on the basis of the robot moving direction angle sequence whether robots serious jitter, when does not appear serious jitter, the APF algorithm is used to calculate the robot move next;

2). if there is a serious jitter, with ANN to predict the trend of the mobile robot, the robot move according to a specified direction and decide whether to reach the target;

3). repeat the process until the robot reaches the target point.

\section{Simulation and Analysis for the Case}

The simulated environment in this work is a two-dimensional plane of 200 meters by 200 meters, in which four areas are square obstacles with a side length of 40 meters. The robot starts from $(0,0)$ with a footstep length of $v=5 m$ and reaches the designated target point $(200,150)$. In order to simplify the demonstration of the case, we only consider the interaction of a mobile robot with the agent, and there are only two types of robots in the case of severe jitter: horizontal right and vertical up. When more complex environments are considered, the movement trend category is increased. Simulation parameters are shown in Table 1.

In this work, TensorFlow [22] is used to build and train the ANN model. The number of sample sets is $2380,80 \%$ of which are training samples, and the remaining $20 \%$ are test samples. The accuracy of the network model obtained from the training set is $97.1 \%$ in the test set. The structural parameters of the ANN model are listed in Table 2.

Figure 6 is the trajectory diagram of the robot using TAPF. It can be observed that when the robot moves between obstacles, the trajectory suffers serious jitter. Figure 7 shows the mobile robot path diagram for the combination of ANN and TAPF. In the design studied, the existence of high moving step and GPS positioning error leads to the randomness of the robot's moving route. Figure 7(a) and Figure 7(b) shows two different movements. Comparing Figure 
6 and Figure 7, it can be observed that the long-term severe jitter does not occur in the moving trajectory by using the proposed scheme.

Table 1. Simulation parameters

\begin{tabular}{ccc}
\hline parameter & state & value \\
\hline$\xi$ & Gravitational gain coefficient & 10 \\
$\eta$ & Repulsive gain coefficient & 2 \\
$d_{0}$ & Range of repulsive field & $20 \mathrm{~m}$ \\
$\beta_{c}$ & Trajectory angle relative difference threshold & $80^{\circ}$ \\
$N$ & Number of historical data & 4 \\
$\mu$ & The mean of GPS positioning error & 0 \\
$\sigma^{2}$ & The variance of GPS positioning error & 10 \\
\hline
\end{tabular}

Table 2. Structure of the artificial neural network

\begin{tabular}{cc}
\hline parameter & value \\
\hline Network layer & 3 \\
Number of neurons in the input layer & 8 \\
Number of hidden layer neurons & 17 \\
Number of neurons in the output layer & 2 \\
The activation function & ReLU function \\
\hline
\end{tabular}

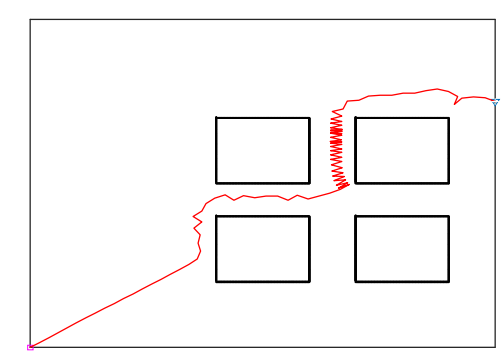

Figure 6. Robot movement trajectory of TAPF method

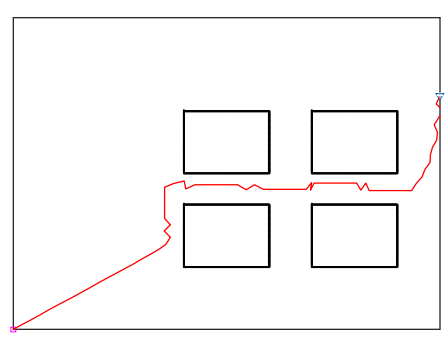

(a) Track one

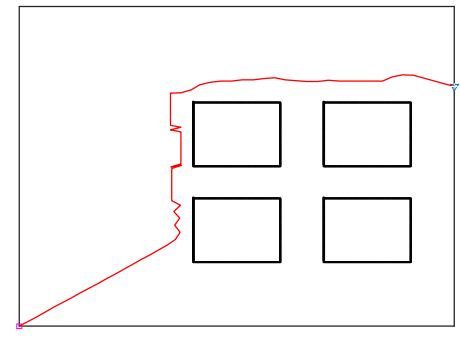

(b) Track two

Figure 7. Moving trajectory of artificial potential field method combined with ANN 
In order to more intuitively reflect the dithering effect of the proposed scheme, Table 3 lists the number of algorithm iterations under the two schemes. By comparison, it can be found that the iteration number of the traditional scheme are 95, while the average iteration number of the proposed scheme are 52 , which is only $54.7 \%$ of the traditional scheme. Therefore, it can be proved that the proposed scheme can accelerate the path planning speed of the robot.

Table 3. Number of algorithm iterations

\begin{tabular}{cc}
\hline scheme & Number of iterations \\
\hline Traditional artificial potential field method & 95 \\
New scheme (track 1) & 56 \\
New scheme (track 2) & 48 \\
\hline
\end{tabular}

\section{Conclusion}

Intelligent transportation systems for robots will become an important part of smart cities in the future. However, to achieve secure navigation, a reliable data analysis solution is required. These solutions not only rely on AI cloud computing, but also require heterogeneous machine behavior data. In this work, we initially discuss the methodology involved in the interaction between machine and machine in machine behavior, and give an interesting and safe navigation application case for the future robot world. In the scene studied, a virtual agent with certain intelligence can help robot when the autonomous navigation of a robot lacking environmental information is in trouble. The intelligence should be implemented by using some machine learning algorithms even under small samples. When a high-speed moving scene and GPS positioning error are taken into account, the traditional artificial potential field method may cause serious jitter of the robot's moving trajectory. A combination of ANN and tradition artificial potential field is proposed to predict the next trend. Preliminary results show that the scheme combined with machine behavior data analysis can effectively curb the trajectory jitter of the robot, so as to achieve a safe and intelligent traffic environment.

\section{References}

1. Rahwan, I; Cebrian, M.; Obradovich, N. Machine behavior. Nature. 2019, 568, 477-486.

2. Ilg, W.; Muhlfriedel, T.; Berns, K. A hybrid learning architecture based on neural networks for adaptive control of a walking machine. Proceedings of International Conference on Robotics and Automation, Albuquerque, NM, USA, 25-25 April 1997; pp. 2626-2631.

3. Gaydashenko, A.; Kudenko, D.; Shpilman, A. A Comparative Evaluation of Machine Learning Methods for Robot Navigation Through Human Crowds. Proceedings of the 2018 17th IEEE International Conference on Machine Learning and Applications (ICMLA), Orlando, FL, USA, $17-$ 20 Dec. 2018; pp. 553-557.

4. Ferdowsi, A.; Challita, U.; Saad, W. Deep Learning for Reliable Mobile Edge Analytics in Intelligent Transportation Systems: An Overview. IEEE Veh. Technol. Mag. 2019, 14, 62-70.

5. Chen, Y.; Zhang, Y.; Maharjan, S. Deep Learning for Secure Mobile Edge Computing. arXiv 2017, arXiv: 1709.08025.

6. Huang, K.; Liu, X.; Fu, S.; Guo, D.; Xu, M. A Lightweight Privacy-Preserving CNN Feature Extraction Framework for Mobile Sensing. IEEE T. Depend. Secure (in press).

7. Khatib, O. Real-time obstacle avoidance for manipulators and mobile robots. Proceedings of the 1985 IEEE International Conference on Robotics and Automation (ICRA), St. Louis, MO, USA, 25-28 March 1985; pp. 500-505.

8. Chen, T.; Zhang, G.; Hu, X.; Xiao, J. Unmanned aerial vehicle route planning method based on a star algorithm. Proceedings of the 2018 13th IEEE Conference on Industrial Electronics and Applications (ICIEA), Wuhan, China, 31 May-2 June 2018; pp. 1510-1514.

9. Jayasree, K. R.; Jayasree, P. R.; Vivek, A. Smoothed RRT techniques for trajectory planning. 
Proceedings of the 2017 International Conference on Technological Advancements in Power and Energy ( TAP Energy), Kollam, India, 21-23 Dec. 2017; pp. 1-8.

10. Muhuri, P. K.; Rauniyar, A. Multi-robot coalition formation problem: Task allocation with adaptive immigrants based genetic algorithms. Proceedings of the 2016 IEEE International Conference on Systems, Man, and Cybernetics (SMC), Budapest, Hungary, 9-12 Oct. 2016; pp. 137-142.

11. Zhang, N.; Zhang, Y.; Ma, C.; Wang, B. Path planning of six-DOF serial robots based on improved artificial potential field method. Proceedings of the 2017 IEEE International Conference on Robotics and Biomimetics (ROBIO), 5-8 Dec. 2017; pp. 617-621.

12. Zhang, Y.; Liu, Z.; Chang, L. A new adaptive artificial potential field and rolling window method for mobile robot path planning. Proceedings of the 2017 29th Chinese Control And Decision Conference (CCDC), Chongqing, China, 28-30 May 2017; pp. 7144-7148.

13. Qian, C.; Qisong Z.; Li, H. Improved artificial potential field method for dynamic target path planning in LBS. Proceedings of the 2018 Chinese Control And Decision Conference (CCDC), Shenyang, China, 9-11 June 2018; pp. 2710-2714.

14. Zhou, L.; Li, W. Adaptive Artificial Potential Field Approach for Obstacle Avoidance Path Planning. Proceedings of the 2014 Seventh International Symposium on Computational Intelligence and Design, Hangzhou, China, 13-14 Dec. 2014; pp. 429-432.

15. Ren, J.; McIsaac, K. A.; Patel, R. V. Modified Newton's method applied to potential field-based navigation for mobile robots. IEEE Trans. Rob. 2006, 22, 384-391.

16. Biswas, K.; Kar, I. On reduction of oscillations in target tracking by artificial potential field method. Proceedings of the 2014 9th International Conference on Industrial and Information Systems (ICIIS), Gwalior, India, 15-17 Dec. 2014; pp. 1-6.

17. Kowdiki, K. H.; Barai, R. K.; Bhattacharya, S. Leader-follower formation control using artificial potential functions: A kinematic approach. Proceedings of the IEEE-International Conference on Advances in Engineering, Science And Management (ICAESM -2012), Nagapattinam, Tamil Nadu, India, 30-31 March 2012; pp. 500-505.

18. Hong, Z. A preliminary study on artificial neural network. Proceedings of the 2011 6th IEEE Joint International Information Technology and Artificial Intelligence Conference, Chongqing, China, 2022 Aug. 2011; pp. 336-338.

19. Mazare, A.; Ionescu, L.; Lita, A.; Serban, G.; Ionut, M. Mobile system with real time route learning using Hardware Artificial Neural Network. Proceedings of the 2015 7th International Conference on Electronics, Computers and Artificial Intelligence (ECAI), Bucharest, Romania, 25-27 June 2015; pp. $45-48$.

20. Dai, X.; Wang, J.; Zhao, J. Research on Multi-Robot Task Allocation Based on BP Neural Network Optimized by Genetic Algorithm. Proceedings of the 2018 5th International Conference on Information Science and Control Engineering (ICISCE), Zhengzhou, China, 20-22 July 2018; pp. 478481.

21. Xu, L.; Wang, L. The research of license plate character recognition based on BP network trained by chaos particle swarm optimization. Proceedings of the 2011 Second International Conference on Mechanic Automation and Control Engineering, Hohhot, China, 15-17 July 2011; pp. 5418-5420.

22. Ertam, F.; Aydın, G. Data classification with deep learning using Tensorflow. Proceedings of the 2017 International Conference on Computer Science and Engineering (UBMK), Antalya, Turkey, 5-8 Oct. 2017; pp. 755-758. 